\section{ANCA-associated vasculitis in scleroderma: a case series of fourteen patients}

\author{
Kimberly P. Liang,' Clement J. Michet ${ }^{2}$ \\ 'Department of Medicine and Division \\ of Rheumatology, University \\ of Pittsburgh Medical Center, Pittsburgh, \\ PA; \\ 2Department of Medicine and Division \\ of Rheumatology, Mayo Clinic and Mayo \\ Graduate School of Medicine, Rochester, \\ MN, USA
}

\section{Abstract}

Antimyeloperoxidase (MP0), perinuclear antineutrophil cytoplasmic antibodies (pANCA), and/or clinically evident vasculitis in patients with scleroderma have been reported only rarely. The clinical significance and prognosis of ANCA-associated vasculitis in systemic sclerosis is uncertain. To report a case and identify the clinical characteristics of scleroderma patients with ANCA-associated vasculitis. Patients with both vasculitis and scleroderma occurring between 1976 to 2006 were identified using an electronic diagnostic index. These diagnoses were confirmed by retrospective review of complete medical records. Clinical features and outcomes recorded included age at vasculitis diagnosis, connective tissue disease (CTD) features, type of scleroderma (limited or diffuse); ANCA serology, vasculitic organ system manifestations; and death. Fourteen cases of scleroderma patients with ANCA-associated and/or small vessel vasculitis were identified. The majority (71\%) were female, with mean age at vasculitis diagnosis 53 years. Seven patients (50\%) had overlap CTD features, and the majority (79\%) had limited variant of scleroderma. All of the 10 patients tested were MPO and pANCA positive. Seven patients (50\%) had glomerulonephritis, 11 (79\%) pulmonary involvement including 3 with pulmonary-renal syndrome, 6 skin purpura, and 5 mononeuritis multiplex and/or peripheral neuropathy. Six patients (43\%) died during followup to 2008 . The presence of pANCA-associated small vessel vasculitis is a rarely reported complication of scleroderma. It occurs most commonly in women with limited scleroderma and most commonly includes pulmonary and/or renal involvement, including severe organ-threatening manifestations and death. Further studies are needed to clarify the role and clinical impact of ANCA in scleroderma patients with and without vasculitis.

\section{Introduction}

It has been suggested that scleroderma is primarily a disease of the vasculature, yet the vascular abnormalities in scleroderma are generally considered noninflammatory. ${ }^{1}$ Indeed, the most frequent vessel histopathology in scleroderma is subintimal proliferation and periadventitial fibrosis. ${ }^{2}$ Although vasculitis is known to occur as a complication of many connective tissue diseases, antimyeloperoxidase (MP0), perinuclear antineutrophil cytoplasmic antibodies (pANCA), and/or clinically evident vasculitis in patients with scleroderma have been reported only rarely. Previous case series have suggested a possible association of vasculitis with the limited or CREST variant of scleroderma and features of Sjogren's syndrome, ${ }^{2}$ though multiple other case reports and series have described vasculitis in the diffuse variant of scleroderma as well. ${ }^{1,-9}$

The clinical significance and prognosis of ANCA-associated vasculitis (AAV) in systemic sclerosis (SSc) is uncertain. Specifically, it is unclear how frequently different vasculitic organ system manifestations occur; whether vasculitis occurs more frequently in patients with limited versus diffuse cutaneous variants of SSc; and what the frequency is of overlap connective tissue disease features including Sjogren's syndrome features and other autoimmune serologic markers in scleroderma patients with vasculitis. The objective of this study was to report a case of a scleroderma patient with pANCA-associated microscopic polyangiitis and to identify the clinical characteristics of scleroderma patients with ANCAassociated vasculitis.

\section{Case Report}

Patient was a 60-year-old woman with a three year history of interstitial lung disease that was originally thought to be a farming related hypersensitivity pneumonitis. A computed tomography (CT) scan revealed basilar fibrosis, septal thickening and patchy alveolar infiltrates. A lung biopsy obtained a year after the onset of symptoms revealed chronic interstitial pneumonitis, fibrosis and rare granulomas. The consulting pathologists concluded the findings were compatible with usual interstitial pneumonitis and chronic hypersensitivity pneumonitis. Treatment included chronic 3-4 L home oxygen, tapered prednisone from 2002-2003, and azathioprine $75 \mathrm{mg}$ daily since December 2005. At that time pulmonary hypertension with pulmonary artery (PA) pressures $\sim 69 \mathrm{~mm}$ Hg was diagnosed by cardiac catheterization. There was no response to nitric oxide testing. Warfarin was started. Her past medical history was notable for Raynaud's phenomenon present for at least seven years as well as a positive antinuclear antibody (ANA) found at
Correspondence: Kimberly P. Liang, Heinz 57 Center, University of Pittsburgh 339 Sixth Avenue, Pittsburgh, PA 15222, USA.

E-mail: liangkp@upmc.edu

Key words: scleroderma, vasculitis, antibodies, antineutrophil cytoplasmic.

Acknowledgment: we gratefully acknowledge Dr. Eric Matteson for his critical review of the manuscript.

Conflict of interest: the authors report no conflicts of interest.

Received for publication: 8 September 2010. Revision received: 22 December 2010. Accepted for publication: 23 December 2010.

This work is licensed under a Creative Commons Attribution 3.0 License (by-nc 3.0).

(C) Copyright K.P. Liang and C.J. Michet., 2011

Licensee PAGEPress, Italy

Rheumatology Reports 2011; 3:e2

doi:10.4081/rr.2011.e2

the time of her pneumonitis diagnosis. Subsequently gastroesophageal reflux disease, dysphagia, xerostomia, photosensitivity, telangiectasias, and oral ulcers appeared. In 2005 limited scleroderma with overlap connective tissue disease features was diagnosed.

In March 2006 the patient developed bilateral foot drop and left median nerve neuropathy. She had had progressive difficulty lifting her feet since November 2005. The week of admission, she had developed burning pain and dysesthesias/paresthesias in the left leg, and then the right leg, as well as paresthesias and weakness of the left first through third fingers. Shortly thereafter, she developed palpable purpura on the distal lower legs (Figure 1).

On physical examination, she was drinking water frequently due to xerostomia; but was not in distress. She had sclerodactyly, a few telangiectasias on her upper lip, palpable purpura lesions on the distal lower extremities, with severe livedo reticularis, varicosities, and scaly hyperpigmented papules on the right forearm and torso. Mucous membranes were dry with tongue fissuring, and there were healing right buccal mucosa oral ulcers. Heart rate and rhythm were regular, with a loud second heart sound. Lung exam revealed bilateral crackles in the lower half lung fields. Abdominal exam was benign. Vascular exam revealed 2/4 edema of the lower extremities to the hips, with mild scleredema of the distal lower extremities. Lower extremity sensation was diminished at the ankles with bilateral foot drop (complete loss of dorsiflexor strength); decreased sensation of the left hand first $3 \frac{1}{2}$ digits with weakness in the median nerve distribution.

Laboratory findings included a markedly 
positive MPO (173.3 EU/mL, normal $\leq 5.0$ $\mathrm{EU} / \mathrm{mL}$ ), positive p-ANCA, and very high rheumatoid factor (RF) $(4760 \mathrm{IU} / \mathrm{mL}$, normal $<15 \mathrm{IU} / \mathrm{mL}$ ). ANA was positive at $2.0 \mathrm{U}$ (normal $\leq 1.0 \mathrm{U}$ ). The following tests and serologies were all negative or unremarkable: total hemolytic complement, C3, C4, SSA, SSB, RNP, Sm, Jo-1, and Scl-70 antibodies, double stranded DNA (dsDNA) antibodies, anti-centromere antibodies, cryoglobulins, hepatitis A, B, and C screen, and HIV screen. Coagulation studies were unremarkable, including negative lupus anticoagulant. Skin biopsies (left forearm and right leg) were suggestive of leukocytoclastic vasculitis, with IgM and complement deposition on immunofluorescence.

Mononeuritis multiplex due to pANCA-associated vasculitis was diagnosed, complicating a diagnosis of limited scleroderma with overlap CTD features. During the subsequent 20day hospital course, she was initially treated with urgent pulse methylprednisolone $1 \mathrm{~g}$ intravenously (IV) daily for three days. While trying to transition on the fourth day to oral prednisone $70 \mathrm{mg}$ daily, her symptoms acutely worsened including development of distal leg skin ulcers; hence, another three-day course of pulse IV methylprednisolone was administered. Oral cyclophosphamide (CYC) therapy was initiated; the dose was gradually increased to $100 \mathrm{mg}(1.3 \mathrm{mg} / \mathrm{kg})$ daily. Oral prednisone with a very gradual taper was initated. She was transferred to the inpatient rehabilitation unit, with good progress during the rehabilitation stay; she had some return of dorsiflexion in the left foot and improved strength in the left first through third fingers, though paresthesias persisted. Pregabalin was started for neuropathic pain, with significant benefit.

After dismissal from the hospital, in followup 3 weeks later, the palpable purpura/skin ulcer lesions were still slow to heal, but she had continued gradual improvement of her neurologic deficits. About 4 weeks post-hospital discharge, she developed leukopenia with white count (WBC) of 2.9 (normal 4.5-11); CYC was held and restarted at $50 \mathrm{mg}$ orally daily several days later. About 7 weeks post-hospital discharge, she then developed acute elevation of liver function tests (with aspartate aminotransferase [AST] and alanine aminotransferase [ALT] over $300 \mathrm{U} / \mathrm{L}$ ) and worsening edema. She was readmitted to the hospital intensive care unit in acute right heart failure from progressive pulmonary HTN; estimated PA pressure 120/36 (systemic BP 99/52). Rapid deterioration followed, and the patient died within 24 hours. Post mortem examination revealed hepatic necrosis with acute, severe venoocclusive disease, severe intimal and medial thickening of small pulmonary arteries and arterioles, but no evidence of active vasculitis. The peripheral nerves were not examined.

\section{Materials and Methods}

\section{Identification of study subjects}

We utilized a centralized electronic diagnostic index to identify patients seen at our institution between 1976 to 2006 with both ANCAassociated vasculitis and scleroderma. Terms used to search the electronic diagnostic index were: "Scleroderma" or "CREST syndrome" with "microscopic polyangiitis" or "polyarteritis nodosa" or "periarteritis nodosa" or "Wegener's granulomatosis." From laboratory data, we also searched for patients who were ANCA positive and anti-centromere and/or Scl70 positive. The electronic searches returned a total of 49 potential cases; however, upon retrospective review of complete medical records to confirm documented clinical diagnoses of both vasculitis and scleroderma, a total of 14 cases were identified.

\section{Data collection}

Through retrospective review of the complete inpatient and outpatient paper and/or electronic medical records, the following clinical features and outcomes were recorded for all 14 identified cases: year/age at diagnosis of vasculitis and diagnosis of scleroderma; presence of overlap connective tissue disease (CTD) features including presence of sicca symptoms; smoking history; type of scleroderma (limited or diffuse); presence of anti-Scl-70 and anticentromere antibodies; presence of ANCA serologies (including pANCA, MPO, cytoplasmic ANCA [cANCA], and proteinase 3 [PR3]); presence of other autoimmune serologies (including ANA, RF, dsDNA, cryoglobulins, SSA, SSB, RNP, Sm, Jo-1); vasculitic organ system manifestations including renal, pulmonary, skin, and neuromuscular involvement; treatments; and mortality.

The presence of overlap CTD features was defined as any physician assessment indicating a clinical impression of "overlap" or "mixed" CTD including features of Sjogren's syndrome, myositis, SLE, and others. Sicca symptoms were recorded as present if there was ever a physician note indicating presence of either xerostomia or xerophthalmia. Smoking history was recorded as never, former (quit $>30$ days prior to vasculitis diagnosis), or current. The categorization of patients as having either limited or diffuse SSc was recorded based on whichever was documented in the medical records, with "CREST syndrome" considered as the limited variant. If the patient had no cutaneous findings of SSc, i.e., "systemic sclerosis sine scleroderma," the patient was classified under limited variant. The presence of ANCA and other autoimmune serologies were all recorded as either positive or negative, defined according to the clinical laboratory reference ranges. Testing was considered positive

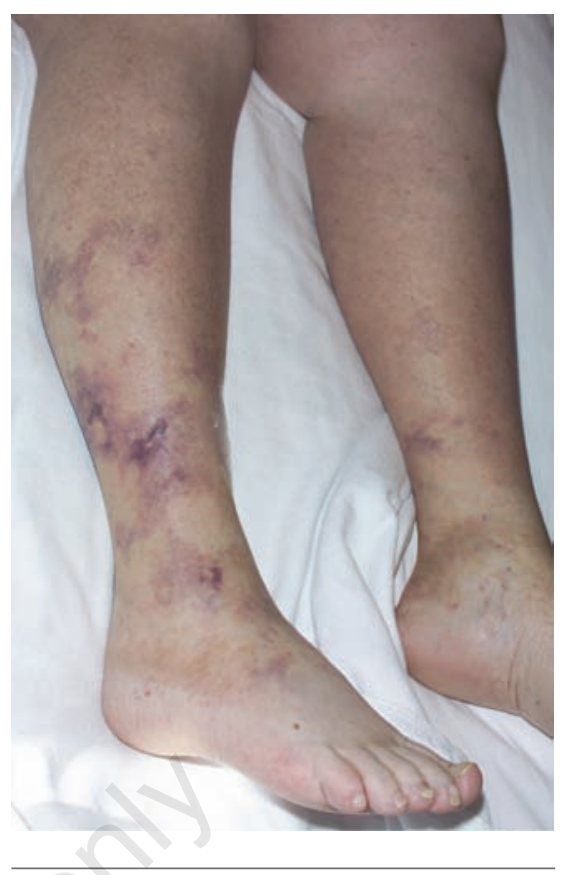

Figure 1. Note the palpable purpura over bilateral distal lower extremities. The patient had complete foot drop of both feet upon presentation.

if there was ever a positive test result.

Renal manifestations recorded were: presence of glomerulonephritis (GN) (based on renal biopsy or well-documented history), end stage renal disease (ESRD), or renal transplantation. Pulmonary manifestations included presence of alveolar hemorrhage (including hemoptysis; clinician diagnosis), interstitial lung disease/pulmonary fibrosis (clinician diagnosis), and pulmonary hypertension (clinician diagnosis based on well-documented history and/or echocardiographic data). Skin involvement was based on clinician diagnoses, presence of purpura and ischemic digital ulcers. Neurologic complications including mononeuritis multiplex and/or peripheral neuropathy, as well as myopathy, were recorded based on clinician diagnoses.

Treatments recorded included use (ever vs. never) of prednisone, CYC, azathioprine (AZA), mycophenolate mofetil (MMF), plasmapheresis (PLEX), angiotensin-converting enzyme inhibitors (ACE), and calcium channel blockers (CCB); as well as ever use of D-penicillamine prior to vasculitis diagnosis. Dates of death or last follow-up were also recorded.

\section{Statistical analysis of data}

Descriptive statistics were used to summarize patient demographics, features of scleroderma diagnosis, ANCA and other autoimmune serologic testing, vasculitic organ system manifestations, treatments, and mortality. 


\section{Results}

\section{Study Subjects}

Forty-nine potential subjects were identified through the diagnostic index search. After medical record review, 14 cases of scleroderma patients with vasculitis were identified. The case report described above was patient \#10.

\section{Demographics and features of scleroderma}

Table 1 summarizes the demographics and features of the scleroderma diagnosis in these patients.

The majority (71\%) of patients were female, and mean age at vasculitis diagnosis was 53 years. The mean time between scleroderma diagnosis and vasculitis diagnosis was 1.5 years, including 3 patients who developed vasculitis preceding the diagnosis of scleroderma (patient \#1, 2, and 8). Six patients (43\%) had overlap or "mixed" CTD features, and nine patients (64\%) had sicca symptoms. At time of vasculitis diagnosis, only 1 patient was a current smoker; 5 were former smokers; and 9 were never smokers. The majority (79\%) had the limited variant of scleroderma. Of note, the limited variant included 3 patients without cutaneous manifestations of scleroderma, i.e. "systemic sclerosis sine scleroderma." Out of 10 patients tested, 4 were anticentromere positive; and out of 10 patients tested, 3 were antiScl70 positive.

\section{ANCA and other serologies}

Table 2 summarizes the results of ANCA and other serologic tests performed in the patients. Out of 10 patients tested, all were MPO and pANCA positive; and out of 7 patients tested, only 1 was PR3 positive but cANCA negative. Out of 12 patients tested, all were negative for SSA or SSB antibodies. Out of 11 patients tested, 5 were RF positive; and out of 13 patients tested, 11 were ANA positive. Double stranded DNA antibodies were undetectable in all of those tested $(n=12)$, as were cryoglobulins $(n=12)$. The majority of antibodies to other extractable nuclear antigens were also undetectable (all negative out of 12 tested for RNP and Sm; and 8 negative out of 9 patients tested for $\mathrm{J}_{0}-1$ ).

\section{Vasculitis organ system involvement}

Table 3 shows the vasculitic organ system manifestations in the 14 cases. All but one (patient \#14) had findings consistent with microscopic polyangiitis. Patient \#14 had MPO and pANCA-positive aortitis with fibrosing mediastinitis. Seven patients (50\%) had GN, and 3 developed ESRD; 2 required renal transplantation. The diagnosis of $\mathrm{GN}$ was made based on renal biopsies in 6 out of the 7 patients, and the remaining one (patient number 9) had a well-documented history of pANCA associated ESRD from GN. Eleven patients (79\%) had pulmonary involvement, including 5 with alveolar hemorrhage, 10 with interstitial lung disease (ILD), and 3 with pulmonary hypertension (HTN). Three patients had pulmonary-renal syndrome. Six patients (40\%) had skin purpura, and 2 had ischemic digital ulcers. Five patients (36\%) had mononeuritis multiplex and/or peripheral neuropathy, and 2 (14\%) had myopathies.

\section{Treatment and survival}

Only one patient had been taking D-penicil- lamine prior to vasculitis diagnosis (patient number 9). All patients were treated with high dose corticosteroids, and six (43\%) were treated with cyclophosphamide (CYC). Six patients (43\%) died during followup to June 2008. The median followup time after the diagnosis of vasculitis was 4.3 years (interquartile range $0.2,9.9$ years).

\section{Discussion}

The presence of ANCA in patients with SSc has been reported to be rare, ranging from about $2.5-9 \%$ of SSc patients. ${ }^{4-5,10-11}$ In one study,

Table 1. Demographics and scleroderma features of patients.

\begin{tabular}{|c|c|c|c|c|c|c|c|}
\hline $\begin{array}{l}\text { Patient } \\
\text { number }\end{array}$ & Gender & $\begin{array}{c}\text { Year of } \\
\text { Scleroderma Dx }\end{array}$ & $\begin{array}{c}\text { Year } \\
\text { of Vasculitis Dx }\end{array}$ & $\begin{array}{l}\text { Age } \\
\text { at Vascullitis Dx }\end{array}$ & $\begin{array}{l}\text { Overlap } \\
\text { CTD }\end{array}$ & $\begin{array}{l}\text { Skin } \\
\text { Subset }\end{array}$ & Serology \\
\hline 1 & $\mathrm{~F}$ & 1969 & 1965 & 62 & + & $\mathrm{DSc}$ & NR \\
\hline 2 & $\mathrm{~F}$ & 1987 & 1979 & 60 & + & LSc & NR \\
\hline 3 & $\mathrm{~F}$ & 1983 & 1986 & 34 & + & DSc & NR \\
\hline 4 & $\mathrm{~F}$ & 1982 & 1990 & 45 & + & LSc & - \\
\hline 5 & M & 1995 & 1996 & 42 & - & LSc & - \\
\hline 6 & $\mathrm{~F}$ & 1999 & 2002 & 65 & - & LSc & +Centro \\
\hline 7 & M & 2000 & 2002 & 61 & - & LSc & - \\
\hline 8 & M & 2004 & 1995 & 51 & - & LSc & +Centro \\
\hline 9 & $\mathrm{~F}$ & 1988 & 2004 & 44 & - & DSc & + Scl-70 \\
\hline 10 & $\mathrm{~F}$ & 2006 & 2006 & 60 & + & LSc & - \\
\hline 11 & $\mathrm{~F}$ & 2002 & 2002 & 69 & - & LSc & +Centro \\
\hline 12 & M & 1996 & 2003 & 46 & + & LSc & +Centro \\
\hline 13 & $\mathrm{~F}$ & 2002 & 2002 & 70 & - & LSc & + Scl-70 \\
\hline 14 & $\mathrm{~F}$ & 2003 & 2003 & 34 & - & LSc & + Scl-70 \\
\hline
\end{tabular}

F, Female; M = Male; Dx, diagnosis; Overlap CTD, overlap or "mixed" connective tissue disease features; DSc, diffuse scleroderma; LSc/CREST, limited scleroderma or CREST syndrome, including systemic sclerosis sine scleroderma (3 patients)*; Centro, anti-centromere antibodies; Scl-70, anti Scl-70 antibodies; NR, not recorded. *Patient \#7: Raynaud's, Esophageal dysmotility consistent with scleroderma; Patient \#8: Raynaud's, digital ischemia, telangiectasia, pulmonary hypertension; Patient \#14: Raynaud's, Esophageal dysmotility and small bowel hypomotility and bacterial overgrowth consistent with scleroderma, positive Scl70 antibody.

Table 2. ANCA and other autoimmune serologies in patients.

\begin{tabular}{lcccccc} 
Patient number & NPO & PANCA & PR3 & CANCA & ANA & RF \\
1 & NR & NR & NR & NR & - & - \\
2 & NR & NR & NR & NR & - & - \\
\hline 3 & NR & NR & NR & NR & + & - \\
4 & NR & NR & NR & NR & + & + \\
\hline 5 & + & + & NR & - & NR & NR \\
6 & + & + & + & - & + & NR \\
\hline 7 & + & + & NR & - & + & - \\
8 & + & + & - & - & + & - \\
\hline 9 & + & + & - & - & + & - \\
10 & + & + & - & - & + & + \\
\hline 11 & + & + & NR & - & + & + \\
12 & + & + & - & - & + & NR \\
\hline 13 & + & + & - & - & + & + \\
14 & + & + & - & - & + & + \\
\hline
\end{tabular}

*MPO, myeloperoxidase antibodies; pANCA, perinuclear antineutrophil cytoplasmic antibodies; PR3, proteinase 3 antibodies; cANCA, cytoplasmic antineutrophil cytoplasmic antibodies; ANA, antinuclear antibodies, including lupus erythematosus (LE) clot test; RF, rheumatoid factor; double stranded DNA antibodies; cryoglobulins; NR, not recorded. 
pANCA presence was found in 6 of $100(6 \%)$ patients with $\mathrm{SSc}$, all with rapidly progressive renal failure without malignant $\mathrm{HTN} .{ }^{4}$ In another study, MPO and pANCA positivity was found in 1 of 11 (9\%) of serum samples from patients with diffuse SSc, who had pitting ulcers and histologically-proven purpuric vasculitis. ${ }^{5}$ Interestingly, as in our case report, that patient was a middle-aged woman with severe and rapidly progressive pulmonary disease with right-sided heart failure, with sparing of the kidneys. ${ }^{5}$ Locke et al. ${ }^{10}$ found MPO antibodies in 2 of 81 (2.5\%) SSc patients' sera, and Ruffatti et al. ${ }^{11}$ found pANCA positivity in 5 of 115 (4.3\%) SSc patients' sera. Interestingly, none of the patients with pANCA positivity in Ruffatti et al.'s study had clinically evident vasculitis, ${ }^{11}$ and the presence of ANCA in SSc is of controversial clinical significance. ${ }^{11,12}$ The purpose of our study was not to examine the clinical significance of ANCA in SSc patients, but rather to better characterize the clinical findings that do occur in SSc patients with clinically evident ANCA-assoiated vasculitis (AAV). There have been numerous case reports and case series describing the presence of AAV in scleroderma, with various organ system manifestations. In our case series, 7 out of the 14 scleroderma patients with AAV presented with GN. ANCA-positive crescentic GN in SSc has recently been recognized to represent a subset of a "different kind of renal crisis" in scleroderma patients, with suggestion that the presence of ANCA be routinely evaluated when faced with renal failure in the setting of SSc. ${ }^{3}$ Multiple case reports and case series support this, with most describing SSc patients with normotensive renal failure found to have MPO and pANCA positivity and pauci-immune necrotizing crescentic GN on renal histopathology. ${ }^{1,3,4,-10,13-26}$ Much of this literature has been reviewed recently. ${ }^{9,18}$ Overall, the vast majority of such patients derived benefit from treatment with high-dose corticosteroids and/or IV or oral CYC.

There has been some speculation in the literature that AAV in scleroderma may be related to exposure to D-penicillamine, as this drug has been associated with pANCA vasculitis. ${ }^{10,14,27,28}$ However, in such cases the patients had clinical improvement after not only Dpenicillamine withdrawal but also concomitant treatment with immunosuppressive agents. In addition, other reports have not corroborated a consistent association with D-penicillamine use, and in our series only one patient had prior D-penicillamine exposure.

There has been recent speculation that preexisting idiopathic pulmonary fibrosis (IPF) may be a consequence of anti-MPO antibody mediated lung damage or potentially contributing factor for the risk of microscopic polyangiitis (MPA) in a subset of IPF patients. ${ }^{29-30}$ The relationship of fibrotic lung damage and MPA requires further investigation. It is notable that two thirds of the patients in our study had scleroderma related intersitial lung disease at the time of vasculitis diagnosis.

Another frequently reported vasculitic organ system manifestation, both in our series and the literature, is pulmonary involvement, ${ }^{1,4,13}$ with generally poor survival especially in those with pulmonary-renal syndrome. ${ }^{4,31}$ In our series, pulmonary involvement was frequent (79\%), including alveolar hemorrhage in 5 patients and pulmonary-renal syndrome in 3 patients, \#2 with limited SSc and 2 of whom died. Hence, it appears that a pulmonary-renal syndrome presentation of microscopic polyangiitis may occur in SSc patients with both limited and diffuse forms of the disease, with poor prognosis. However, in both the patient reported by Wutzl et al., ${ }^{1}$ as well as patient \#10 in our series, treatment with high dose corticosteroids and cyclophosphamide allowed for survival despite pulmonary-renal syndrome.

Less prevalent in the literature are reports of skin and neuromuscular involvement as vasculitic organ system manifestations in SSc patients with AAV. Only the case series by Oddis et al. ${ }^{2}$ suggested that vasculitis presented primarily as cutaneous lesions with ulceration and/or mononeuritis multiplex, as in our case report. This may be in part because the subset of SSc patients with normotensive renal failure due to pANCA-positive crescentic GN has only been recognized more recently, since about 1994 . Herrick et al..$^{32}$ reported that 5 out of 9 patients with severe digital ischemia from SSc (all of the limited variant) had histologic evidence of vasculitis. In our current series of 15 cases, we found 6 had skin purpura, 2 had ischemic digital ulcers, 5 had mononeuritis multiplex and/or peripheral neuropathy, and 2 had myopathies. Hence, these manifestations of AAV do occur in SSc patients, but in a minority.

Vasculitis in SSc has been found to be associated with Sjogren's syndrome and the CREST syndrome variant. ${ }^{2}$ In addition, Casari et al. ${ }^{17}$ reported that out of a case series of $5 \mathrm{SSc}$ patients with pANCA positivity, 3 had "overlap" CTD features, including 2 with polymyositis (PM) features and 1 with SLE features. Interestingly, in our series about half had sicca symptoms, but none had SSA or SSB out of those tested $(n=13)$. More definitive testing

Table 3. Vasculitic organ system manifestations of patients.

\begin{tabular}{|c|c|c|c|c|c|c|c|c|c|}
\hline \multirow[t]{2}{*}{ Patient number } & \multicolumn{3}{|c|}{ Renal } & \multicolumn{3}{|c|}{ Pulmonary } & \multicolumn{2}{|c|}{ Skin } & Neuromuscular \\
\hline & GN & ESRD & $\begin{array}{l}\text { Renal } \\
\text { Txpt }\end{array}$ & $\begin{array}{c}\text { Alveolar } \\
\text { Hem }\end{array}$ & $\begin{array}{l}\text { ILD/Pulm } \\
\text { Fibrosis }\end{array}$ & $\begin{array}{l}\text { Pulm } \\
\text { HTN }\end{array}$ & Purpura & $\begin{array}{l}\text { Ischemic } \\
\text { digital ulcers }\end{array}$ & Neuro Myopathy \\
\hline
\end{tabular}

\begin{tabular}{|c|c|c|c|c|c|c|c|c|c|c|}
\hline 1 & - & - & - & - & + & - & - & - & + & + \\
\hline 2 & + & - & - & + & - & - & + & - & - & - \\
\hline 3 & - & - & - & - & - & - & + & - & - & - \\
\hline 4 & - & - & - & - & + & - & + & - & - & + \\
\hline 5 & + & - & - & - & + & - & - & - & - & - \\
\hline 6 & + & + & - & + & + & - & - & - & + & - \\
\hline 7 & - & - & - & + & + & - & - & - & - & - \\
\hline 8 & + & + & + & - & - & + & - & + & - & - \\
\hline 9 & + & - & - & + & + & - & - & + & - & - \\
\hline 10 & - & - & - & - & + & + & + & - & + & - \\
\hline 11 & - & - & - & + & + & - & + & - & + & - \\
\hline 12 & + & + & + & - & + & + & - & - & - & - \\
\hline 13 & + & - & - & - & + & - & + & - & + & - \\
\hline
\end{tabular}

$\begin{array}{llllll}14 & - & - & - & - & -\end{array}-$ including mononeuritis multiplex and/or peripheral neuropathy. 
(e.g., lip biopsies, salivary scintigraphy or sialograms) to diagnose Sjogren's were not performed, as sicca symptoms are known to be a common clinical feature of scleroderma. Our results also support a possible association between vasculitis and limited or CREST variant of SSc, present in 11 (79\%) patients. Furthermore, in our series about half had features of overlap or "mixed" CTD. Besides sicca symptoms, most frequently the overlap CTD features in our series included systemic lupus erythematosus (SLE) features.

Of further interest, in our series 3 patients had no cutaneous manifestations of SSc, i.e. "systemic sclerosis sine scleroderma." Their specific characteristics are provided in Table 1. Tomioka et al..$^{18}$ and Katrib et al..$^{16}$ also reported cases of patients with "systemic sclerosis sine scleroderma" who developed ANCA-related crescentic GN, presenting with normotensive renal failure. We suggest that vigilance for small vessel vasculitic complications should be maintained particularly in patients with the limited or CREST variant of scleroderma, especially those with sicca symptoms; as well as patients with "systemic sclerosis sine scleroderma" and overlap or "mixed" CTD with predominant sclerodermatous features.

The presence of PR3 and cANCA and/or Wegener's granulomatosus in patients with SSc has been reported exceedingly rarely. ${ }^{33,34}$ Our results support the exceedingly rare presence of cANCA in SSc patients with vasculitis, with only one patient with positive PR3 but negative cANCA, a false positive result. The vast majority of SSc patients with vasculitis present with MPO and pANCA-positive microscopic polyangiitis. Only one of our patients had a pANCA-positive large vessel vasculitis (aortitis with fibrosing mediastinitis). It should be noted that the presence of ANCA antibodies alone in patients with scleroderma is not always associated with clinical vasculitis. PANCA/MPO antibodies have been found in up to $6 \%$ of scleroderma patients screened serologically. With follow up vasculitis may occur in a small number of those positive. ${ }^{35}$

All of our patients were treated with high dose corticosteroids and $40 \%$ were treated with CYC, with at least modest clinical improvements, though mortality was still $40 \%$ with median follow-up time of 4.3 years. As discussed above, the literature to date supports a beneficial effect of corticosteroids and/or CYC, particularly for pANCA-associated crescentic GN and pulmonary-renal syndrome. Prognosis is worse in those patients with pulmonaryrenal syndrome. ${ }^{4}$ Morbidity (including treatment-related) and mortality of these patients in comparison to other patients with SSc is uncertain.

Limitations of our study include its retrospective nature, the lack of an inception cohort of scleroderma patients, non-uniformity of cli- nician testing for ANCA and availability of ANCA testing over time, variability of medical record-keeping over time, and possibility of referral bias in cases seen at our institution. Strengths of our study include careful and complete ascertainment of cases through the use of electronic diagnostic index, thorough chart review of scleroderma, CTD and vasculitis features, and the number of cases in our series (one of the largest to date).

In conclusion, the presence of pANCA-associated vasculitis is a rarely reported complication of scleroderma. It occurs most commonly in women with limited or CREST variant of scleroderma, as well as those with sicca symptoms and/or "overlap" CTD features. Vasculitic organ system manifestations most frequently include pulmonary and/or renal involvement. Severe manifestations including pulmonaryrenal syndrome and death may occur, and treatment with high dose corticosteroids and cyclophosphamide appears to afford benefit. The presence of cANCA is exceedingly rare in scleroderma patients with clinically evident vasculitis. Further studies are needed to compare the prognosis of these patients to other scleroderma patients without vasculitis.

\section{References}

1. Wutzl AL, Foley RN, O'Driscoll BR, et al. Microscopic polyangiitis presenting as pulmonary-renal syndrome in a patient with long-standing diffuse cutaneous systemic sclerosis and antibodies to myeloperoxidase. Arthritis Rheum 2001; 45:533-6.

2. Oddis CV, Eisenbeis CH, Jr., Reidbord HE, et al. Vasculitis in systemic sclerosis: association with Sjogren's syndrome and the CREST syndrome variant. J Rheumatol 1987;14:942-8.

3. Kamen DL, Wigley FM, Brown AN. Antineutrophil cytoplasmic antibody-positive crescentic glomerulonephritis in scleroderma--a different kind of renal crisis. $\mathrm{J}$ Rheumatol 2006;33:1886-8.

4. Endo H, Hosono T, Kondo H. Antineutrophil cytoplasmic autoantibodies in 6 patients with renal failure and systemic sclerosis. J Rheumatol 1994;21:86470 .

5. Kiraz S, Simsek H, Ertenli I, et al. Antineutrophil cytoplasmic antibodies in systemic sclerosis. Clin Rheumatol 1996;15:519-20.

6. Herrera-Esparza R, Aguilar JL, Saucedo A, et al. Scleroderma with type III glomerulonephritis and MPO-ANCA antibodies in the serum. J Eur Acad Dermatol Venereol 2005;19:617-20.

7. Carvajal I, Bernis C, Sanz P, et al.
Antineutrophil cytoplasmic autoantibodies (ANCA) and systemic sclerosis. Nephrol Dial Transplant 1997;12:576-7.

8. Omote A, Muramatsu M, Sugimoto Y, et al. Myeloperoxidase-specific anti-neutrophil cytoplasmic autoantibodies -- related scleroderma renal crisis treated with doublefiltration plasmapheresis. Intern Med 1997;36:508-13.

9. Cheung G, Chew G, Wyndham R, et al. Myeloperoxidase-antineutrophil cytoplasmic antibody seroconversion and fulminant vasculitis in Scl-70-positive scleroderma. Intern Med J 2007;37:205-7.

10. Locke IC, Worrall JG, Leaker B, et al. Autoantibodies to myeloperoxidase in systemic sclerosis. J Rheumatol 1997;24:86-9.

11. Ruffatti A, Sinico RA, Radice A, et al. Autoantibodies to proteinase 3 and myeloperoxidase in systemic sclerosis. $\mathrm{J}$ Rheumatol 2002;29:918-23.

12. Hoffman GS, Specks U. Antineutrophil cytoplasmic antibodies. Arthritis Rheum 1998;41:1521-37.

13. Akimoto $\mathrm{S}$, Ishikawa 0 , Tamura T, Miyachi Y. Antineutrophil cytoplasmic autoantibodies in patients with systemic sclerosis. $\mathrm{Br}$ J Dermatol 1996;134:407-10.

14. Hillis GS, Khan IH, Simpson JG, Rees AJ. Scleroderma, D-penicillamine treatment, and progressive renal failure associated with positive antimyeloperoxidase antineutrophil cytoplasmic antibodies. Am J Kidney Dis 1997;30:279-81.

15. Villaverde V, Balsa A, Cabezas JA, et al. Normotensive renal failure in a patient with systemic sclerosis and p-antineutrophil cytoplasmic autoantibodies which developed into Paget's disease of bone after immunosuppressive therapy. Rheumatology (Oxford) 1999;38:190-1.

16. Katrib A, Sturgess A, Bertouch JV. Systemic sclerosis and antineutrophil cytoplasmic autoantibody-associated renal failure. Rheumatol Int 1999;19:61-3.

17. Casari S, Haeney M, Farrand S, Herrick A. Antineutrophil cytoplasmic antibodies a "Red Flag" in patients with systemic sclerosis. J Rheumatol 2002;29:2666-7.

18. Tomioka M, Hinoshita F, Miyauchi N, et al. ANCA-related crescentic glomerulonephritis in a patient with scleroderma without marked dermatological change and malignant hypertension. Intern Med 2004;43: 496-502.

19. Rho YH, Choi SJ, Lee YH, et al. Scleroderma associated with ANCA-associated vasculitis. Rheumatol Int 2006; 26:465-8.

20. Anders HJ, Wiebecke B, Haedecke C, et al. MPO-ANCA-Positive crescentic glomerulonephritis: a distinct entity of scleroderma renal disease? Am J Kidney Dis 1999;33:e3. 
21. Arnaud L, Huart A, Plaisier E, et al. ANCArelated crescentic glomerulonephritis in systemic sclerosis: revisiting the "normotensive scleroderma renal crisis". Clin Nephrol 2007;68:165-70.

22. Harashima S, Yoshizawa S, Horiuchi T, et al. [A case of systemic sclerosis with crescentic glomerulonephritis associated with perinuclear-antineutrophil cytoplasmic antibody (p-ANCA)]. Nihon Rinsho Meneki Gakkai Kaishi 1999;22:86-92.

23. Kobayashi M, Saito M, Minoshima S, et al. [A case of progressive systemic sclerosis with crescentic glomerulonephritis associated with myeloperoxidase-antineutrophil cytoplasmic antibody (MPO-ANCA) and anti-glomerular basement membrane antibody (anti-GBM Ab)]. Nippon Jinzo Gakkai Shi 1995;37:207-11.

24. Martinez Ara J, Picazo ML, Torre A, et al. [Progressive systemic sclerosis associated with anti-myeloperoxidase ANCA vasculitis with renal and cutaneous involvement]. Nefrologia 2000;20:383-6.

25. Mizutani A, Tanaka I, Katayama M, et al. [A case of myeloperoxidase-antineutrophil cytoplasmic antibody (MPO-ANCA) related glomerulonephritis associated with systemic sclerosis treated by steroid pulse therapy: a case report]. Ryumachi 2000;40: 828-32.

26. Maes B, Van Mieghem A, Messiaen T, et al. Limited cutaneous systemic sclerosis associated with MPO-ANCA positive renal small vessel vasculitis of the microscopic polyangiitis type. Am J Kidney Dis 2000; 36:E16.

27. Gaskin G, Thompson EM, Pusey CD. Goodpasture-like syndrome associated with anti-myeloperoxidase antibodies following penicillamine treatment. Nephrol Dial Transplant 1995;10:1925-8.

28. Vazquez-Del Mercado M, Mendoza-Topete A, Best-Aguilera CR, Garcia-De La Torre I. Diffuse alveolar hemorrhage in limited cutaneous systemic sclerosis with positive perinuclear antineutrophil cytoplasmic antibodies. J Rheumatol 1996;23:1821-3.

29. Foulon G, Delaval P, Valeyre D, et al. ANCAassociated lung fibrosis: analysis of 17 patients. Respir Med 2008;102:1392-8.

30. Tzelepis GE, Kokosi M, Tzioufas A, et al. Prevalence and outcome of pulmonary fibrosis in microscopic polyangiitis. Eur
Respir J 2010;36:116-21.

31. Helfrich DJ, Banner B, Steen VD, Medsger TA, Jr. Normotensive renal failure in systemic sclerosis. Arthritis Rheum 1989;32: 1128-34.

32. Herrick AL, Oogarah PK, Freemont AJ, et al. Vasculitis in patients with systemic sclerosis and severe digital ischaemia requiring amputation. Ann Rheum Dis 1994;53:323-6.

33. Caramaschi P, Biasi D, Tonolli E, et al. Antineutrophil cytoplasmic antibodies in scleroderma patients: first report of a case with anti-proteinase 3 antibodies and review of the literature. Joint Bone Spine 2002;69:177-80.

34. Huong DL, Papo T, Gatfosse M, et al. Antineutrophil cytoplasmic autoantibodies in systemic sclerosis with renal failure. J Rheumatol 1995;22:791-2.

35. Ramos-Casals M, Nardi N, Brito-Zeron P, et al. Atypical autoantibodies in patients with primary Sjogren syndrome: clinical characteristics and follow-up of 82 cases. Semin Arthritis Rheum 2006;35:312-21. 